\title{
Hemobilia secundaria a aneurisma de la arteria hepática
}

\section{Hemobilia Secondary to Aneurysm of the Hepatic Artery}

Pablo Galindo, MD, ${ }^{1}$ Julián David Martínez, MD, ${ }^{2}$ Mario Humberto Rey, MD. ${ }^{3}$

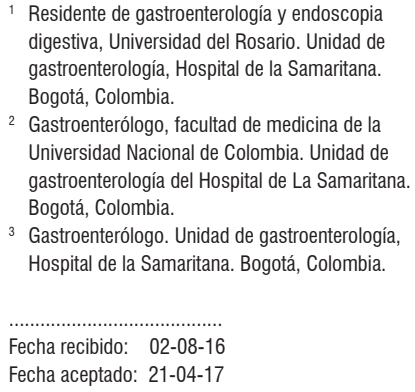
digestiva, Universidad del Rosario. Unidad de gastroenterología, Hospital de la Samaritana. Bogotá, Colombia.

2 Gastroenterólogo, facultad de medicina de la Universidad Nacional de Colombia. Unidad de gastroenterología del Hospital de La Samaritana. Bogotá, Colombia.

${ }^{3}$ Gastroenterólogo. Unidad de gastroenterología, Hospital de la Samaritana. Bogotá, Colombia.

Fecha recibido: $\quad 02-08-16$ Fecha aceptado: $21-04-17$

\author{
Resumen \\ Se presenta el caso de un paciente con hemorragia de vías digestivas altas e ictericia asociadas a un aneu- \\ risma de la arteria hepática derecha. \\ Palabras clave \\ Hemobilia, hemorragias de vías digestivas altas, aneurisma de arteria hepática.

\section{Abstract} \\ We present the case of a patient with upper digestive tract hemorrhaging and jaundice due to an aneurysm \\ of the right hepatic artery.

\section{Keywords} \\ Hemobilia, hemorrhages of high digestive tract, hepatic artery aneurysm.
}

\section{INTRODUCCIÓN}

La hemobilia es la presencia de sangre en la vía biliar y es una causa rara de hemorragia digestiva, es poco considerada y, por tanto, es poco diagnosticada.

El primer caso fue descrito por Francis Glisson en 1654. Quinke, en 1871, describió un caso de hemobilia con sus características clínicas que son la clásica tríada: ictericia, dolor en el hipocondrio derecho y hemorragia digestiva alta.(1-3). Kehr, en 1903, practicó la primera cirugía de un paciente con hemobilia secundaria a ruptura de un aneurisma de la arteria hepática (AAH) (4).

Las causas de hemobilia son variadas, dentro de las cuales se destacan las traumáticas, iatrogénicas (manipulación quirúrgica o endoscópica de la vía biliar), inflamatorias, infecciosas, neoplásicas y vasculares (aneurismas, vasculitis y malformaciones arteriovenosas).
El AAH tiene una prevalencia estimada del 0,4\% del total de los aneurismas y representa casi una quinta parte de todos los aneurismas de las arterias viscerales (3-5). Su incidencia ha venido en aumento en los últimos años, probablemente debido a la disponibilidad de mejores imágenes diagnósticas (6). Existen múltiples etiologías de $\mathrm{AAH}$, tales como la ateroesclerosis, enfermedades autoinmunes con compromiso vascular (panarteritis nudosa, lupus eritematoso sistémico, arteritis de Takayasu, entre otras), síndrome de Marfan, tuberculosis y la instrumentación quirúrgica o endoscópica de la vía biliar. La mayoría de estos aneurismas se localizan en el hilio hepático y con menor frecuencia a nivel intrahepático. La mayor parte de los pacientes permanecen asintomáticos; sin embargo, se pueden presentar con dolor abdominal, sangrado digestivo por hemobilia y choque hipovolémico. Cuando hay ruptura aneurismática, la mortalidad estimada es del $40 \%$ de los casos (5-9). 
Presentamos el caso de un paciente con hemorragia de vías digestivas altas e ictericia por la presencia de un aneurisma de la arteria hepática derecha.

\section{CASO CLÍNICO}

Hombre de 38 años, con antecedente de déficit cognitivo severo por síndrome convulsivo crónico y esquizofrenia paranoide, sin otros antecedentes patológicos, quirúrgicos o de trauma. Remitido por cuadro de 15 días de evolución con hematemesis, deposiciones melénicas, ictericia mucocutánea y dolor en el hipocondrio derecho. En el centro de remisión realizaron una esofagogastroduodenoscopia, que mostró sangre fresca a nivel duodenal sin precisarse su origen; y una colonoileoscopia, que no evidenció lesiones que explicaran el sangrado; por tanto, le fue practicado un estudio con videocápsula endoscópica, cuyo resultado fue normal. Presentó un sangrado digestivo intrahospitalario con descenso de hemoglobina hasta $6,8 \mathrm{~g} / \mathrm{dL}$, fue transfundido con 2 unidades de glóbulos rojos empaquetados $y$, ulteriormente, remitido a nuestra institución. Al ingreso se encontró con ictericia generalizada, hemodinámicamente estable con dolor a la palpación del hipocondrio derecho y el tacto rectal mostró presencia de melenas.

Los principales resultados de laboratorio fueron: hemoglobina de $8,9 \mathrm{~g} / \mathrm{dL}$, volumen corpuscular medio de $72 \mathrm{ft}$, bilirrubina total de $6 \mathrm{mg} / \mathrm{dL}$ y directa de $4,3 \mathrm{mg} / \mathrm{dL}$, fosfatasa alcalina de $1067 \mathrm{UI} / \mathrm{L}$. La endoscopia de vías digestivas altas mostró un sangrado activo en capa proveniente de la ampolla de Vater, por lo que se sospecha hemobilia. En el ultrasonido abdominal se observó una lesión vascular en el hilio hepático sugestiva de dilatación aneurismática. Se realizó un estudio de angiografía por tomografía axial computarizada (TAC) abdominal, que demostró una dilatación aneurismática de $37 \times 39 \times 34 \mathrm{~mm}$ con trombo del 50\% en su interior, en estrecha relación con vesícula biliar y, al parecer, dependiente de la arteria hepática derecha. Por los hallazgos descritos, se practicó una arteriografía selectiva; se observó un aneurisma de la arteria hepática derecha con las dimensiones descritas anteriormente y con un trombo en su interior. Se realizó una embolización y se logró el implante de 3 microcoils de forma selectiva con cierre completo del cuello aneurismático. La TAC abdominal demostró una disminución del tamaño de la lesión hasta 19 x $18 \mathrm{x}$ $15 \mathrm{~mm} 5$ días después; sin embargo, llenado con el medio de contraste de la cavidad aneurismática y turbulencia en su interior. En una nueva arteriografía y embolización con microcoils se logró la exclusión completa del aneurisma con oclusión distal y proximal del mismo (Figura 1).

$\mathrm{Su}$ evolución clínica fue favorable, desapareció el dolor abdominal, resolvió su ictericia y no volvió a presentar sangrado gastrointestinal luego del procedimiento.

\section{DISCUSIÓN}

La hemobilia es una causa de hemorragia digestiva alta rara y es aún menos diagnosticada debido a que se requiere una alta sospecha clínica y una gran experiencia del personal
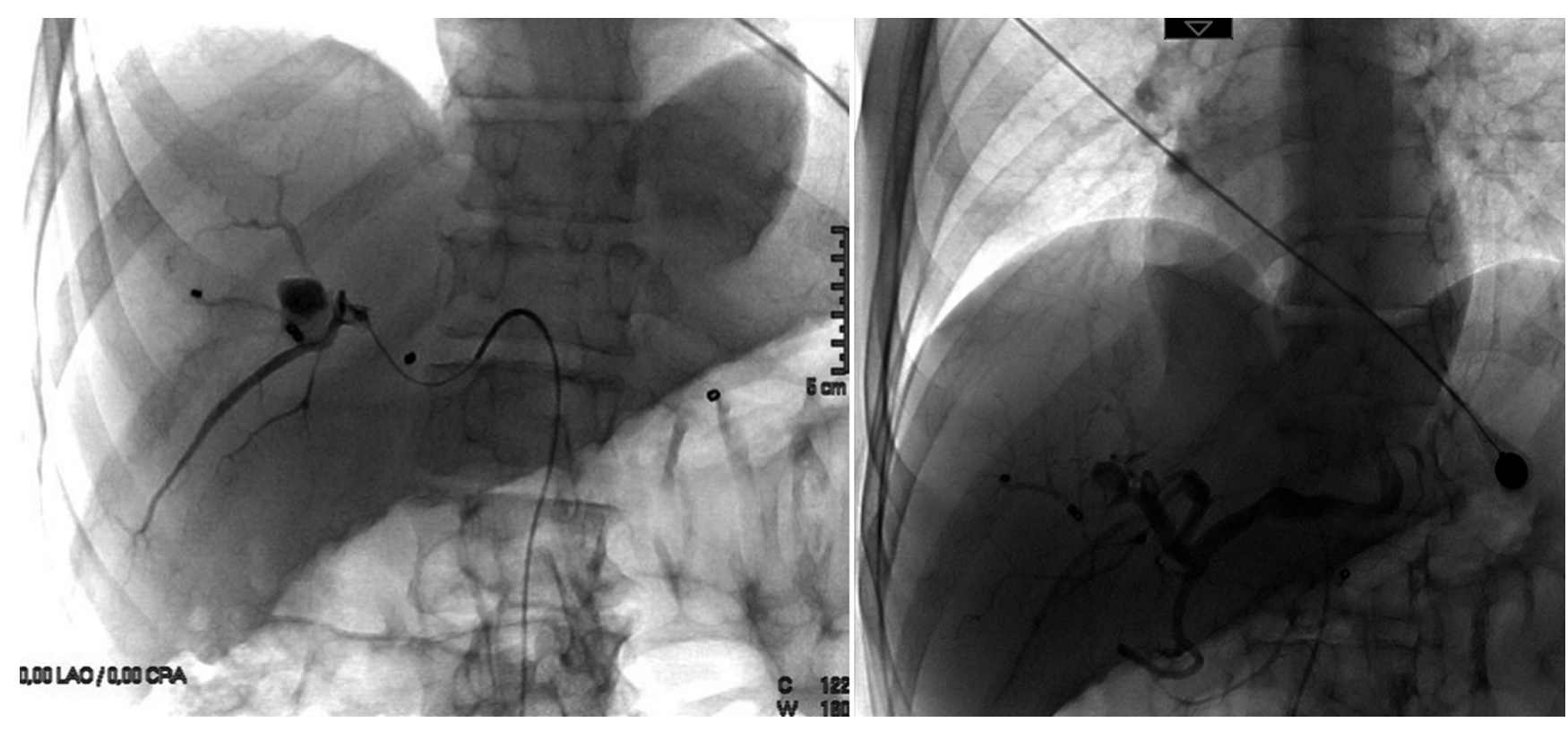

Figura 1. Arteriografía y embolización de aneurisma de arteria hepática derecha. 
médico que atiende estos pacientes. Su incidencia exacta se desconoce, pero se ha descrito una mortalidad muy alta, de hasta el 25\% (10). En este caso, se resalta la importancia de sospechar esta patología en aquellos pacientes que se presentan con sangrado digestivo oscuro manifiesto (11).

El paciente informado presentó los signos clínicos clásicos de hemobilia (dolor en el hipocondrio derecho, ictericia y evidencia de hemorragia digestiva alta), tal como sucede en cerca del $40 \%$ de los afectados; los restantes casos no presentan esta tríada.

Como se describe en la literatura médica, el diagnóstico inicial de hemobilia es escurridizo y en la mayoría de los pacientes se requiere de la práctica de varios procedimientos endoscópicos por diferentes observadores sin que se logre, en la mayoría de casos, llegar al diagnóstico; lo que hace recomendable que en los pacientes con sangrado digestivo oscuro su reevaluación clínica y endoscópica deba ser realizada por médicos con amplia experiencia en la atención de las urgencias gastroenterológicas.

La esofagogastroduodenoscopia es el método diagnóstico de elección porque, además de descartar otras causas de hemorragia, puede demostrar el flujo de sangre proveniente de la papila duodenal (12). Los estudios diagnósticos complementarios son la ultrasonografía (USG) abdominal, TAC abdominal contrastada, resonancia magnética nuclear (RMN) de abdomen y arteriografía selectiva.

Los AAH son una causa extremadamente rara de ictericia obstructiva y hemobilia. Son infrecuentemente diagnosticados, pero su incidencia ha ido en aumento gracias al desarrollo de mejores técnicas de imágenes. En la mayoría de los casos, los AAH son asintomáticos y cuando se presentan con síntomas se asocian con un mal pronóstico si el diagnóstico se retrasa.

La hemorragia de vías digestivas alta (HVDA) o hemobilia secundaria a AAH justifican estrategias de manejo urgentes puesto que la ruptura puede ser fatal. Existen múltiples estudios complementarios para el diagnóstico de la entidad: La angiografía por TAC permite la detección con buena confiabilidad, aunque la angiografía hepática sigue siendo el estándar de oro dentro de las opciones diagnósticas y terapéuticas con la embolización transarterial selectiva, como la primera elección de manejo con tasas de éxito el $80 \%-100 \%$ y menor mortalidad, en comparación con procedimientos quirúrgicos $(4,5)$. Otras opciones terapéuticas incluyen la reparación quirúrgica e implante de stents, las cuales quedarían reservadas cuando no es posible la embolización transarterial (5).

\section{CONCLUSIÓN}

La hemobilia siempre debe sospecharse en los pacientes con evidencia de sangre en el duodeno y especialmente en aquellos que se presentan con la tríada clásica. El AAH es una de las condiciones que puede causar hemobilia y, de no considerarse, puede no ser diagnosticado. La angiografía hepática sigue siendo el estándar de oro para el diagnóstico y el abordaje terapéutico en estos casos.

\section{REFERENCIAS}

1. Sandblom P. Hemobilia (biliary tract hemorrhage): history, pathology, diagnosis, treatment. Springfield: Charles C. Thomas; 1972.

2. Merrell S, Schneider P. Conferences and review. Hemobilia, evolution of current diagnosis and treatment. West J Med. 1991;155(6):621-5.

3. Petrou A, Brennan N, Soonawalla Z, et al. Hemobilia due to cystic artery stump pseudoaneurysm following laparoscopic cholecystectomy: case presentation and literature review. Int Surg. 2012;97(2):140-4. Doi: https://doi.org/10.9738/CC52.1

4. Beltrán F, Castillo A, Hidalgo H. Hemobilia secundaria a pseudoaneurisma roto: reporte de caso. Rev Gastroenterol Peru. 2015;35(1):89-92.

5. Panno C, Gutiérrez S, Echeverría R, et al. Hemobilia secundaria a pseudoaneurisma de la arteria hepática. Acta Gastroenterol Latinoam. 2015;45(2):133-6.

6. Peter G, Shaheer R, Narayanan P, et al. Hepatic artery aneurysm: a rare case of obstructive jaundice with severe hemobilia. Ann Gastroenterol. 2014;27(3):288-9.

7. Suarez Grau JM, Rubio Chaves C, Pareja Ciuro F, et al. Obstructive jaundice secondary to hepatic artery aneurysm. Cir Cir. 2008;76(3):253-6.

8. García C, Pérez Ramírez P, Martorell-Lossius A, et al. Aneurismas de las arterias viscerales. Cir Esp. 2005;78(4):24650. Doi: https://doi.org/10.1016/S0009-739X(05)70926-X

9. Alhawsawi A, Aljiffry M, Walsh M, et al. Hepatic artery aneurysm associated whith prune belly syndrome: a case report and review of the literature. J Surg Educ. 2009;66(1):43-7. Doi: https://doi.org/10.1016/j.jsurg.2008.10.004

10. Baillie J. Hemobilia. Gastroenterol Hepatol (N Y). 2012;8(4):270-2.

11. Gerson LB, Fidler JL, Cave DR, et al. Clinical guideline: diagnosis and management of small bowel bleeding. Am J Gastroenterol 2015;110(9):1265-87. Doi: https://doi. org/10.1038/ajg.2015.246

12. Rencuzogullari A, Okoh AK, Akcam TA, et al. Hemobilia as a result of right hepatic artery pseudoaneurysm rupture: An unusual complication of laparoscopic cholecystectomy. Int J Surg Case Rep. 2014;5(3):142-4. Doi: https://doi. org/10.1016/j.ijscr.2014.01.005 